\title{
The human microbiome: Role in health and disease and its potential applications
}

\author{
Jennifer Perera ${ }^{1}$ \\ ${ }^{1}$ Faculty of Medicine, University of Colombo, Sri Lanka
}

\begin{abstract}
Article Information
Total number of

Words 3633

Author has no conflicts of interest to declare

Keywords: Human microbiome, Health, Disease.

Date of submission: 16.01 .2018

Date of acceptance: 25.01 .2018

DOI:

http://doi.org/10.4038/cjms.v54i2.4927

\author{
Author responsible for \\ correspondence: \\ Jennifer Perera \\ Dean and Chair Professor of \\ Microbiology \\ Faculty of Medicine, \\ University of Colombo \\ P O Box 271, Colombo \\ Sri Lanka
}

http://orcid.org/0000-0002-7450-5672

Abstract

The human microbiota comprises all microorganisms living within the human body and microbiome is their collective genome. They include eukaryotes, archaea, bacteria and viruses. Most microbes live in the gut, particularly in the large intestine. The number of genes contributed to by the microbes is 100 times the number of genes in the human genome. The sheer microbial abundance suggests that the human body is a collection of human and microbial cells and their genes resulting in a blend of human and microbial traits.

The microbiome is essential for maintenance of human life. The microbes that live in and on us are mostly beneficial colonizers and have many important functions such as assisting in digestion, regulating immune system, producing essential vitamins and protecting against bacteria that cause diseases. Research has demonstrated that dysbioses in the human microbiome correlate with numerous disease states, including inflammatory bowel disease, malignancy and autoimmune diseases.
\end{abstract}

\section{Introduction}

The human body is colonized by an array of microorganisms, which outnumber the host cells by about 10 to 1 . The microbial community, which comprises 10 - 100 trillion symbiotic microbial cells, is collectively known as the 'human microbiota' and the collection of the microbial genome is termed as 'human microbiome'[1]. The human microbiome is also known as our "forgotten organ"[2].
Therefore, the humans have two genomes, the genome inherited from the parents which is almost stable and the acquired microbiome which is extremely dynamic. Although humans are 99\% identical to each other in their genetic make-up, they are significantly different from one another in terms of the human microbiome [1].

The microbes which inhabit the human body are important for human physiology, immune system development, digestion and detoxification of harmful substances. 
Some of these microorganisms residing in the gut encode proteins involved in functions required for host survival, but not found in the human genome. They produce enzymes required for the hydrolysis of specific indigestible compounds in the diet, and the synthesis of vitamins [3].

A variety of microorganisms usually colonize the surfaces of the human body which are exposed to the external environment. This diverse array of microorganisms which inhabit humans include bacteria, archaea, viruses and eukaryotic microorganisms with the dominant phyla being Bacteroidetes and Firmicutes [4]. Several research studies have emphasized the difference in compositions and diversity of the human microbial community in different body sites [5-7]. It was also identified that every individual's habitat is characterized by a few signature species. A significantly high degree of diversity has been observed in gut and mouth compared to other regions of the body [8].

Although the variation of microbial community within a subject over time is relatively low at each body site, there is also considerable inter-individual variability $[9,10]$. From the point of view on health and disease, understanding the microbiota variations between individuals is of utmost importance in the study of its impact on understanding disease conditions. Among the multiple factors that contribute to the shaping of the host microbiome, environmental factors such as exposure to cigarette smoke, air pollutants, allergens and antibiotics play a major role [11-14]. Host associated factors such as age, gender, genetics, diet, medications, are also vital determinants of adult microbiome $[15,16]$.

The microbes that inhabit humans have dynamic interactions with the environment, and are freely transferred between different sites. Although there is considerable inter-individual variability in taxonomic composition of microbes, the metabolic pathways in a specific body site are considerably stable among individuals. This highlights the fact that the human microbiome has shared "core" of functionalities [2].

New advancements in microbiology such as cultureindependent high-throughput sequencing and metabolomics studies are aimed at determining the relationship between the microbiome and the disease conditions and targeting therapies against individual patient's microbiome rather than targeting the relatively stable host genome [1].

It has long been speculated, that relationships between humans and their microbiota are beneficial in maintaining health. Yet, at the same time the same microbiota has been implicated in the aetiology of various diseases in humans. In this Review, I give an overview of the current understanding of the microbiota and its different interactions in healthy individuals. I will then go on to discuss the imbalances in the composition of the microbiota, and relate to diseases such as obesity, diabetes etc. and argue that functional studies rather than composition based studies will foster a deeper understanding of the many diseases and lead to microbiota based therapeutic options.

\section{Role of Human microbiota in maintenance of health}

\section{Role in metabolism}

Endogenous human gut microbiota plays a major role in nutrient metabolism. Main source of nutrients to gut microbiota is derived by fermentation of carbohydrates which are otherwise indigestible by the host $[3,6]$. The colonic organisms such as Bacteroides, Roseburia, Bifidobacterium, Fecalibacterium, and Enterobacteriaare mainly responsible for this function whichresult in the synthesis of short chain fatty acids such as butyrate, propionate and acetate, which are rich sources of energy for the host $[17,18]$. Thus, gut microorganisms enhance the ability of humans to extract energy from diverse diets. Short chain fatty acids are also important for modulating immune response [3]. Human gut microbiota is also involved in the synthesis of several important compounds such as Vitamin K and some components of Vitamin B, linoleic acid, secondary bile acids [19]. Gut microbiota has the ability to suppress the inhibition of lipoprotein lipase activity in adipocytes and thereby impart a positive effect on lipid metabolism [20]. The gut microbiota is also enriched with an efficient protein metabolizing machinery that function through the microbial proteinases and peptidases in partnership with human proteinases [21].

\section{Role in immunity}

The role of gut microbiota in regulation of immune system and immunomodulation has been extensively studied. This involvement is identified with regards to both innate and adaptive immune systems [22,23]. Studies comparing normal mice with germ free (GF) mice have found that GF mice show extensive defects in the development of gut-associated lymphoid tissue and antibody production $[24,25]$.

Humans are continuously exposed to a number of xenobiotics (e.g. drugs, drug metabolites, environmental pollutants) in day-to-day life. A 
convincing body of evidence has described the role of human microbiome in detoxification of xenobiotics. This is mainly achieved by inducing gut microbiota to express genes which are capable of metabolizing xenobiotics [26].

\section{Potential impact of microbiome on diseases}

\section{Role in gastrointestinal diseases}

Human gut is home for about 100 trillion microorganisms. The three main dominant phyla found in gut are namely, Firmicutes, Bacteroidetes and Actinobacteria [27]. The composition varies significantly along the gastrointestinal tract and a progressive increase in both density and diversity is observed in distal segments.

Gut microbiota plays an important role in maintenance of a healthy immune system, by aiding in development and maturation of the immune system. As the gastrointestinal tract is continuously exposed to foreign substances, this ability of immune system to identify pathogens and mount an appropriate response is of paramount importance. Inability to do so may result in uncontrolled inflammation and development of conditions such as inflammatory bowel disease [28].

The rise in the incidence of inflammatory disorders in the last decade has been attributed to modifications in gut microbial community. Loss of bacterial diversity and microbial dysbiosis are two major concerns in patients with this disease. Changes in microbial conformation, inappropriate immune response towards commensal microbial antigens are some of the abnormalities identified in the pathogenesis of inflammatory bowel disease. For instance, an increase in Bacteroidetes and Proteobacteria coupled by a decrease in Firmicutes, with an overall reduction in diversity is reported in Crohn disease. A significant reduction in the population of Faecalibacterium prausnitzii, a Firmicute with anti-inflammatory properties was also identified in these studies [29]. Dysbiosis of intestinal microbiota has been implicated in the pathogenesis of Ulcerative colitis too, but to a lesser extent [30].

As described earlier, gut microbiome is able to digest material like plant polysaccharides, which are contained in dietary fibre. Short chain fatty acid, butyrate produced in this process, has important anti-inflammatory activities in addition to providing energy for colonic epithelial cells [31]. This has been implicated in the pathogenesis of inflammatory bowel disease and other intestinal diseases [32].

Use of probiotics is a treatment option in patients with inflammatory bowel disease. Probiotics are beneficial microbes that imparts a positive impact on the gut microbial composition and function [33]. Data from the results of clinical trials suggest that probiotics consisting of lactic acid bacteria may be effective in treatment of ulcerative colitis [34]. and to a lesser extent in Crohn disease [35].

\section{Role in gastrointestinal malignancy}

Aside from the widely accepted genetic factors, nongenetic factors including residential microbes in the gut has an impact on development of cancers in the gastrointestinal tract. Helicobacter pylori associated chronic inflammation is considered the strongest risk factor for development of gastric cancer and elimination of the $H$ pylori prior to development of gastric atrophy may protect against development of cancer in the stomach [36]. Similarly a pathological imbalance of the microbial flora has been observed in patients with adenomas compared to normal controls [37]. Although results have been variable, Pseudomonas, Helicobacter and Acinetobacterspp are found in higher numbers with lower abundance of butyrate producing protective bacteria [38].

Moreover, many other bacteria derived metabolites have been implicated both in the suppression and colon cancer development [39]. It has been suspected for a long time that disturbance of microbiota is also associated with colorectal cancer [40]. Experimental studies conducted using colitis susceptible IL-10- mouse strains, demonstrated that bacteria specific factors may be involved in the development of colitis associated colorectal cancer [41]. Chronic inflammation also plays an important role in pathogenesis of colorectal cancer, possibly by creating a platform for bacteria to adhere to colonic mucosa and thus altering the colonic microbial composition. In addition, inflammation has an effect on gene expression and may influence functional capabilities of microbes [42].

Genetic studies have been able to identify specific microbial groups associated with colorectal cancer. For example, analysis of stool samples of patients diagnosed with colorectal cancer, have shown to harbour anaerobic bacterial group BacteroidesPrevotella in abundance, compared to healthy individuals [43]. Bioactive substances produced by intestinal microbiota perform many functions like degrading protective mucins and activating environmental carcinogens [43]. These may influence the occurrence of colorectal cancer.

Another fact that supports the link between colonic microbiota and colorectal cancer is the ability of antibiotics to alter the microbial composition and affect the expression of host genes involved in the 
pathogenesis of colorectal carcinoma [44].

\section{Role in obesity and type 2 diabetes mellitus}

A marked increase in incidence and prevalence of obesity is observed, due to the sedentary life style, genetic and other environmental factors within the community. Additive to these novel factors, recent studies have revealed the significant role of intestinal microbiome in precipitation of obesity within a population [44]. It is interesting to note that studies carried out in mice as well as humans have shown that gut microbiota differ in their composition between obese and lean subjects $[45,46]$.

Further research carried out to identify the association of intestinal microbiome and occurrence of obesity, have shown that the changes occurring in gut microbiome related to the composition and metabolic function leads to extraction of increased amounts of calories from the diet [47]. This additionally extracted energy gets deposited in the adipose tissue leading to obesity of the individual.

Under normal circumstances, the energy storage and utilization is determined by the interaction of intestinal microorganisms with host epithelial cells. Yet due to increased age and dietary malpractices, the composition of normal gut microbiome has changed markedly, leading to increased absorption of calories. Increased intake of high fat diet has shown to affect the intestine in two main ways. Compared with their lean controls, obese humans showed a decrease in Bacteroidetes and a corresponding increase in Firmicutes, the two main phyla in the gastro intestinal tract [48]. Secondly, it leads to disruption of epithelial integrity leading to changes in cellular permeability. All these ultimately lead to obesity of the individual.

Type 2 diabetes mellitus is the most common form of disease accounting for approximately $90 \%$ of cases. It is a disease characterized by the reduced sensitivity of receptors to insulin, despite the presence of sufficient insulin within the circulation. Research evidence suggests that the microbial flora in intestines play a role in regulation of insulin sensitivity. Some research studies suggest that irrespective of the obesity status of the individual, type 2 diabetes mellitus itself cause changes in the composition of gut microbiome [44].

Studies on obesity have also shown that obese individuals have altered small chain fatty acid production in comparison to normal individuals. Due to this abnormality, obese individuals are subjected to impairment in satiety, hepatic glucose and lipid production as well as inflammation. As an added function, butyrate, which is produced in the digestion of dietary fibre, prevents translocation of endotoxic compounds. In the absence of sufficient butyrate, translocation of endotoxins occur leading to low grade inflammation, which in turn has an impact on insulin resistance and occurrence of type 2 diabetes mellitus [49]. Therefore all the research findings support the concept that occurrence of obesity and type 2 diabetes mellitus have an association with changes in intestinal microbiome.

\section{Role in lung diseases}

The lung, which contains numerous bronchioles, alveolar sacs is known to be a sterile area without any microorganisms. Contrary to this, recent research studies have discovered presence of bacterial DNA, fungi and viruses beyond the level of terminal bronchioles in individuals who have no specific respiratory infections [50]. Thus, the concept of healthy microbiome in lungs has become a widely accepted fact.

Density of lung microbiome is mainly determined by entry of organisms to the lower airways through inhalation, disruption of preventive barriers leading to reduced elimination of organisms from airways and environmental factors with which the person lives with. Under normal circumstances the entry and elimination is in a balanced state thus the density of microorganisms is usually static [51-53].

When compared to gut microbiome, the lung microbiome is more variable and dynamic due to the bidirectional air flow through the airways. In terms of bacterial density of airways, it is comparable to that of the duodenum [54]. Differences in the anatomical structures like the epithelial surfaces and the variances in the functional aspects like production of mucous, differentiate the gut and lung community of microorganisms in a broader manner.

Role of microbiome in lungs ranges from maintenance of normal health of the individual, prevention of diseases and initiation and mediation of metabolic pathways in response to injurious agents. Asthma is a chronic airway disease with reversible airflow limitation. Microbiome plays an important role in the occurrence of allergic asthma $[55,56]$. It is mainly a consequence of activation and maturation of innate and adaptive immune systems in the body. Exposure to specific microoraganisms is a key factor in early onset asthma. Research evidence suggests that harbouring Moraxella catarrhalis and Haemophilus influenzae in the hypopharynx of infants implicate an additional risk on the child for development of asthma [57]. Furthermore, adult asthmatics have found to harbour an increased amount of proteobacteria in lower 
airway tract secretions compared to healthy individuals [58].

Chronic obstructive pulmonary disease (COPD) has an insidious onset and progresses over time with acute intermittent exacerbations. COPD patient's microbiome largely differs from normal microbiome due the immune cell infiltration and corresponding inflammatory reactions. There is a reduction in the diversity of respiratory microbiome in COPD patients [59]. Although the microbial community harbouring the respiratory tract of COPD patients is subjected to continuous change, $H$ influenzae, $M$. catarrhalis and $S$. pneumoniae are identified as the most prominent microoraginisms in the stable state of COPD. Acute bacterial exacerbations of COPD was dominated by the phyla Proteobacteria (mainly Haemophilus spp.). Firmicutes were the dominant figures in eosinophilic exacerbations [60].

\section{Role in skin diseases}

The skin is home to a diverse microbial community [61]. Colonization of microbes is determined by the topographical location, host factors and environmental factors. Actinobacteria, Proteobacteria, Firmicutes, and Bacteroidetes are the most dominant phyla of bacteria that inhabit the skin [62]. Topographical variation is a key feature observed in skin microbiota. The regions which are high in temperature, humidity and moisture favour growth of microflora such as Staphylococcus and Corynebacterium spp whereas dry areas of the skin harbour greater amounts of Proteobacteria and Bacteroidetes $[63,64]$. Sebaceous areas tend to be harboured by high proportions of bacterium Propionibacterium spp [63-65].

Certain dermatological conditions manifest at stereotypical sites. For instance, psoriasis manifests on extensor surfaces of elbow whereas atopic dermatitis manifests on the flexor surfaces [66]. Characterizing the microbiota that colonize specific sites may thus provide an insight to understanding the pathophysiological basis of dermatological conditions.

Acne vulgaris is a common skin condition most prevalent among adolescents. Although the aetiology is still unclear, researchers have identified that Gram-positive lipophilic anaerobe Propionibacterium acnes play a role in acne pathogenesis. Pilosebaceous units in skin matures at onset of puberty and provides optimum atmosphere for lipophilic microorganisms. Acne commonly occurs in sites with high density of sebaceous glands as these sites favour growth of lipophilic microorganisms. Due to the bacterial involvement in pathogenesis of acne, antibiotics are a wellestablished therapeutic modality [66].
A link between skin microbiome changes and progress of disease is identified in atopic dermatitis, which is a chronic inflammatory skin condition commonly seen in childhood. In the majority of patients suffering from atopic dermatitis, the skin becomes colonized by $S$. aureus [67]. These species can penetrate the epidermis and induce an inflammatory response. $S$. aureus is also capable of inducing disease flares [68]. Use of dilute bleach baths with intermittent intranasal application of mupirocin ointment decreased the clinical severity of atopic dermatitis and provides supporting evidence [69].

Hence, a thorough understanding of the microbial communities and their variations would be of utmost importance in managing many dermatological conditions.

\section{Role in psychiatric diseases}

The presence of a gut-brain axis play a key role in maintaining normal brain function and this has been known for decades [70]. This axis has been extended to the "microbiota-gut-brain axis," and all these organ systems are in constant interactions [70]. Gut microbiota dysbiosis is known to lead to the translocation of bacteria through gut wall and into the mesenteric lymphoid tissue, which in turn provokes an immune response. The resulting release of inflammatory cytokines cause activation of vagus and spinal afferent neurons [71,72]. Autism spectrum disorder (ASD) has been reported as correlated with an altered gut microbiota. The low relative abundances of the mucolytic bacteria Akkermansiamuciniphila and Bifidobacterium spp. have been found in the faeces of children with autism [73]. Similarly, faecal microbiotic composition has increased levels of Enterobacteriaceae and Alistipes, but reduced levels of Faecalibacteriumin patients with major depressive disorder [74]. These studies suggest that the role of the gut microbiota needs further investigation in psychiatric diseases.

\section{Advances in research and applications}

\section{Metabolomics}

A review on microbiome is incomplete with no reference to metabolomics. Microbiome studies focus on composition or answer the question "What is there?". In contrast the metabolomics study the function or answer the question "What are they doing?. In other words, metabolomics is the study of host - microbial interactions including exchange of metabolic products and signalling molecules, which in turn provides the essential functional role to the host and microorganisms. Metabolic biomarkers have been developed to assess these functionalities. 
The quantitative measurement of these metabolites using mass spectrometry will characterise a particular phenotype of the microbiome [75].

\section{Advancements in microbial technology}

During the past two decades microbiome research has been revolutionised by high throughput sequencing technology and bioinformatics analysis. Only $50 \%$ of the gut microbes that are identified by $16 \mathrm{~S}$ rDNA high throughput sequencing are cultivable even using gnotobiotic mice and anaerobic culture systems [76]. It is also widely known that the microbes with the highest abundance are not always the most active ones. Therefore, to achieve precise microbiome related applications in medicine it is necessary to distinguish microbes that mediate essential host microbiome interactions from the rest.

Numerous chip based isolation devices (egiChip) have been developed which has hundreds of miniature diffusion chambers, each of which can be inoculated with a single cell and recovery rates are known to be higher compared to conventional culture techniques [77]. An advancement of this technique is the iTip which traps the microbe in a gel, that allows the study of metabolites and nutrients [78]. There are many other developments such as the 'Simulator of the Human Intestinal Microbial Ecosystem' colonic stem cell constructions, microfluidic devices that have been used to study the microbe host interactions in detail [79].

\section{Future directions and Applications}

Human microbiome, our "second genome" plays a role on genetic diversity, disease modification and immunity and its functional component can influence metabolism. With advances in technology leading to better research outcomes, microbiome analysis of an individual would be useful to diagnose and predict diseases [80]. Prevention and treatment of diseases by targeting the microbiome is being widely investigated and some treatment modalities are already in application for $C$. difficle infections [81] and liver diseases [82]. Future research should be focussed at a deeper level, on providing evidence of causality and mechanisms of microbe human interactions, made possible with ever-expanding novel technologies and approaches with a view to developing therapeutic applications.

\section{Acknowledgements:}

I thank Dr. Krishna Ranathunga for conducting the literature search.

\section{References}

1. Ursell LK, Metcalf JL, Parfrey LW, et al. Defining the human microbiome. Nutr Rev 2012;70(suppl1):S38-44.

2. D'Argenio V, Salvatore F. The role of the gut microbiome in the healthy adult status. ClinicaChimicaActa2015;451:97-102.

3. Flint HJ, Scott KP, Duncan SH, et al. Microbial degradation of complex carbohydrates in the gut. Gut Microbes 2012;3:289-306.

4. U Wollina. Microbiome in atopic dermatitis. ClinCosmetInvestigDermatol2017;10:51-56.

5. Shukla SD, Budden KF, Neal R, et al. Microbiome effects on immunity, health and disease in the lung. Clinical and Translational Immunology 2017;6(3):e133.

6. Relman DA. The human microbiome: ecosystem resilience and health. Nutr Rev 2012;70(s1).

7. Jandhyala SM, Talukdar R, Subramanyam C, et al. Role of the normal gut microbiota. World $J$ Gastroenterol 2015;21(29):8787-8803.

8. Caporaso JG, et al. Moving pictures of the human microbiome. Genome Biol 2011;12(5):R50.

9. Costello, EK, Lauber, CL, Hamady, M, et al. Bacterial community variation in human body habitats across space and time. Science 2009;326:1694-97.

10. Robinson, CJ, Bohannan, BJ, and Young, VB. From structure to function: the ecology of hostassociated microbial communities. MicrobiolMolBiol Rev. 2010;74:453-76.

11. Turnbaugh, PJ, Ridaura, VK, Faith, JJ, et al. The effect of diet on the human gut microbiome: a metagenomic analysis in humanized gnotobiotic mice. SciTransl Med 2009;1(6):6ra14

12. Dethlefsen L, Huse S, Sogin ML, et al The pervasive effects of an antibiotic on the human gut microbiota, as revealed by deep $16 \mathrm{~S}$ rRNA sequencing. PLoSBiol 2008; 6: 280.

13. Jernberg C, Lo“fmark S, Edlund C, et al. Long term ecological impacts of antibiotic administration on the human intestinal micro- biota. ISME J 2007; 1:56-66.

14. Sommer MO, Dantas G, Churc GM. Functional characteriza-tion of the antibiotic resistance reservoir in the human microflora. Science2009;325:1128-1131.

15. Muegge BD, Kuczynski J, Knights D, et al. Diet drives convergence in gut microbiome functions across mammalian phylogeny and within humans. Science 2011;332(6032):970-974.

16. Hollister EB, Gao C, Versalovic J. Compositional and functional features of the gastrointestinal microbiome and their effects on human health. Gastroenterology 2014;146(6):1449-58.

17. Macfarlane S, Macfarlane GT. Regulation of shortchain fatty acid production. Proc NutrSoc 2003;62:67-72.

18. Sartor RB. Microbial influences in inflammatory bowel diseases. Gastroenterology. 2008;134:577- 
594.

19. Kang Z, Zhang J, Zhou J, et al. Recent advances in microbi- al production of $\delta$-aminolevulinic acid and vitamin B12. BiotechnolAdv 2012;30(6):1533-42.

20. Hooper LV, Wong MH, Thelin A, et al. Molecular analysis of commensal host-microbial relationships in the intestine. Science2001;291:881-884.

21. De Biase D, Pennacchietti E. Glutamate decarboxylase-dependent acid resistance in orally acquired bacteria: function, distribution and biomedical implications of the gadBC operon. MolMicrobiol2012;86:770-786.

22. Cebra JJ. Influences of microbiota on intestinal immune system development. Am J ClinNutr1999;69(5):1046S-51S.

23. Thaiss CA, Zmora N, Levy M, et al. The microbiome and innate immunity. Nature 2016;535(7610):65-74.

24. Round JL, Mazmanian SK. The gut microbiota shapes intestinal immune re- sponses during health and disease. Nat Rev Immunol 2009;9(5):313-23.

25.Madsen KL, Doyle JS, Jewell LD, et al. Lactobacillus species prevents colitis in interleukin 10 gene-deficient mice. Gastroenterology 1999;116(5):1107-14.

26. Lu K, Mahbub R, Fox JG. Xenobiotics: interaction with the intestinal microflora. ILAR J 2015;56(2):218-27.

27. Gagnière J, Raisch J, VeziantJ,et al. Gut microbiota imbalance and colorectal cancer. World $J$ Gastroenterol 2016; 22(2):501-18.

28. Rapozo DC, Bernardazzi C, de Souza HS. Diet and microbiota in inflammatory bowel disease: The gut in disharmony. World J Gastroenterol 2017;23(12):2124-2140.

29. Takahashi K, Nishida A, Fujimoto T, et al. Reduced Abundance of Butyrate-Producing Bacteria Species in the Fecal Microbial Community in Crohn's Disease. Digestion 2016;93:59-65.

30. Andoh A, Imaeda H, Aomatsu T, et al. Comparison of the fecal microbiota profiles between ulcerative colitis and Crohn's disease using terminal restriction fragment length polymorphism analysis. J Gastroenterol 2011;46:479-486.

31. Hamer HM, Jonkers D, Venema K, et al. Review article: the role of butyrate on colonic function. Aliment PharmacolTher 2008;27:104119.

32. Pacheco RG, Esposito CC, Müller LC, et al. Use of butyrate or glutamine in enema solution reduces inflammation and fibrosis in experimental diversion colitis. World J Gastroenterol2012;18:4278-4287.

33. Sartor RB, Muehlbauer M. Microbial host interactions in IBD: implications for pathogenesis and therapy. CurrGastroenterol Rep 2007;9:497507.

34. Sartor RB. Therapeutic manipulation of the enteric microflora in inflammatory bowel diseases: antibiotics, probiotics, and prebiotics. Gastroenterology 2004;126:1620-1633.

35. Ewaschuk JB, Dieleman LA. Probiotics and prebiotics in chronic inflammatory bowel diseases. World J Gastroenterol 2006;12:59415950 .

36. Wong BCY, Lam SK, Wong WM, et al. China Gastric Cancer Study Group. Helicobacter pylori eradication to prevent gastric cancer in a high-risk region of China: a randomized controlled trial. JAMA 2004;291(2):187-94.

37. Sanapareddy N, Legge RM, Jovov B, et al. Increased rectal microbial richness is associated with the presence of colorectal adenomas in humans. ISME J 2012; 6(10):1858-68.

38. Zackular JP, Baxter NT, Iverson KD, et al. The gut microbiome modulates colon tumorigenesis. MBio 2013;4(6):e00692-13.

39. Castellarin M, Warren RL, Freeman JD, et al. Fusobacterium nucleatum infection is prevalent in human colorectal carcinoma. Genome Res 2012; 22(2):299-306.

40. Cho I, Blaser MJ. The human microbiome: at the interface of health and disease. Nature Reviews. Genetics $2012 ; 13(4): 260-70$.

41. Hold GL, Smith M, Grange C, et al. Role of the gut microbiota in inflammatory bowel disease pathogenesis: what have we learnt in the past 10 years?. World J Gastroenterol 2014;20(5):11921210.

42. Arthur JC, Perez-Chanona E, Mühlbauer M, et al. Intestinal inflammation targets cancer-inducing activity of the microbiota. Science 2012;338(6103):120-3.

43. Arthur JC, Jobin C. The complex interplay between inflammation, the microbiota and colorectal cancer. Gut Microbes 2013 ;4(3):253-8.

44. Hartstra AV, Bouter KE, Bäckhed F, et al. Insights into the role of the microbiome in obesity and type 2 diabetes. Diabetes Care 2015;38(1):159-65.

45.Ley RE, Bäckhed F, Turnbaugh P, Lozupone $\mathrm{CA}$, et al. Obesity alters gut microbial ecology. Proc Natl AcadSci2005;102:1107011075 .

46. Turnbaugh PJ, Ley RE, Mahowald MA, et al. An obesity-associated gut microbiome with increased capacity for energy harvest. Nature 2006;444:1027-1031.

47.Tilg H, Kaser A. Gut microbiome, obesity, and metabolic dysfunction. Journal Clinical Invest 2011;121(6):2126-2132.

48. Ley RE, Turnbaugh PJ, Klein S, et al. Microbial ecology: human gut microbes associated with obesity. Nature2006;444:1022-1023.

49. Wellen KE, Hotamisligil GS. Inflammation, stress, and diabetes. J Clin Invest2005;115:11111119.

50.Dickson RP, Huffnagle GB. The lung microbiome: new principles for respiratory bacteriology in health and disease. PLoS Pathogens 2015 ;11(7):e1004923.

51.Dickson RP, Erb-Downward JR, Freeman CM, et al. Spatial variation in the healthy human lung microbiome and the adapted island model of lung biogeography. Ann Am ThoracSoc 2015;12(6):82130

52.Dickson RP, Erb-Downward JR, Huffnagle GB 


\section{Perera J.}

Towards an ecology of the lung: new conceptual models of pulmonary microbiology and pneumonia pathogenesis. Lancet Respir Med2014;2:238-246.

53.Venkataraman A, Bassis CM, Beck JM, et al. Application of a neutral community model to assess structuring of the human lung microbiome. MBio 2015 ;6(1):e02284-14.

54. Hilty M, Burke C, Pedro H,et al. Disordered microbial communities in asthmatic airways. PLoS One 2010;5:e8578

55.Hansbro PM, Beagley KW, Horvat JC, et al. Role of atypical bacterial infection of the lung in predisposition/protection of asthma. PharmacolTher 2004;101:193-210.

56. Starkey MR, Essilfie AT, Horvat JC, et al. Constitutive production of IL-13 promotes earlylife Chlamydia respiratory infection and allergic airway disease. Mucosal Immunol 2013;6:569-579.

57. Bisgaard H, Hermansen MN, Buchvald F, et al. Childhood asthma after bacterial colonization of the airway in neonates. $N$ Engl $J$ Med2007;357:1487-1495.

58. Marri PR, Stern DA, Wright AL, et al. Asthmaassociated differences in microbial composition of induced sputum. J Allergy ClinImmunol 2013;131:346-352.

59. Sze MA, Dimitriu PA, Hayashi S, et al. The lung tissue microbiome in chronic obstructive pulmonary disease. Am J RespirCrit Care Med 2012;185:1073-1080.

60. Wang Z, Bafadhel M, Haldar K, et al. Lung microbiome dynamics in COPD exacerbations. EurRespir J 2016;47:1082-1092.

61. Grice EA. The skin microbiome: potential for novel diagnostic and therapeutic approaches to cutaneous disease. SeminCutan Med Surg 2014;33(2):98-103.

62. Hannigan GD, Grice EA. Microbial ecology of the skin in the era of metagenomics and molecular microbiology. Perspect Med 2013;3(12):a015362

63. Grice EA, Kong HH, Conlan S, et al. Topographical and temporal diversity of the human skin microbiome. Science 2009 ;324(5931):1190-2.

64. Costello EK, Lauber CL, Hamady M, et al. Bacterial community variation in human body habitats across space and time. Science 2009;326(5960):1694-1697.

65. Human Microbiome Project Consortium. Structure, function and diversity of the healthy human microbiome. Nature 2012;486(7402):207-214.

66. Leyden JJ, McGinley KJ, Mills OH, et al. Propionibacterium levels in patients with and without acne vulgaris. J Invest Dermatol 1975;65(4):382-384.

67. Wollina U. Microbiome in atopic dermatitis. ClinCosmetInvestigDermatol2017;10:51-56.

68. Shaw TE, Currie GP, Koudelka CW, et al. Eczema prevalence in the United States: data from the 2003 National Survey of Children's Health. J Invest Dermatol 2011;131(1):67-73.

69. Huang JT, Abrams M, Tlougan B, et al. Treatment of Staphylococcus aureus colonization in atopic dermatitis decreases disease severity. Paediatrics 2009;123(5):e808-814

70. Kendler K S. What psychiatric genetics has taught us about the nature of the psychiatric illness and what is left to learn. Mol

Psychiatry2013;18(10):1058-66.

71. Cryan JF, Dinan TG. Mind-altering microorganisms: the impact of the gut microbiota on brain and behaviour. Nature Rev Neurosci2012;13(10):701-12.

72. Maes M, Kubera M, Leunis JC, et al. In depression, bacterial translocation may drive inflammatory responses, oxidative and nitrosative stress (O\&NS), and autoimmune responses directed against O\&NS-damaged neoepitopes. ActaPsychiatrScand 2013;127(5):344-54

73. Wang L, Christophersen CT, SorichMJ, et al. Low relative abundances of the mucolytic bacterium Akkermansiamuciniphila and Bifidobacterium spp. in faeces of children with autism. Appl Environ Microbiol2011; 77(18):6718-21.

74. Jiang H, Ling Z, Zhang Y, et al. Altered fecal microbiota composition in patients with major depressive disorder. Brain BehavImmun 2015;48:186-94.

75. Nicholson, J. K., and Lindon, J. C. Systems biology: metabolomics. Nature 2008;455: 10541056.

76. Arnold JW, Roach J, Azcarate-Peril MA. Emerging technologies for gut microbiome research. Trends Microbiol2016;24(11):887-901.

77. Nichols D, Cahoon N, Trakhtenberg EM, et al. Use of ichip for high-throughput in situ cultivation of "uncultivable" microbial species. Appl Environ Microbiol 2010;76(8):2445-50.

78. Jung D, Seo EY, Epstein SS, et al. Application of a new cultivation technology, I-tip, for studying microbial diversity in fresh- water sponges of Lake Baikal, Russia. FEMS MicrobiolEcol 2014;90(2):417- 23.

79. Baohong Wang, Mingfei Yao, LongxianLv, et al. The Human Microbiota in Health and Disease. Engineering 2017;3(1):71-82.

80. Lu H, Zhang C, Qian G, et al. An analysis of microbiota- targeted therapies in patients with avian influenza virus subtype H7N9 infection. BMC Infect Dis2014;14:359.

81.van Nood E, Vrieze A, Nieuwdorp M, et al. Duodenal infusion of donor feces for recurrent Clostridium difficile. $N$ Engl J Med2013;368(5):407-15.

82. Dhiman RK, Rana B, Agrawal S, et al. Probiotic VSL\#3 reduces liver disease severity and hospitalization in patients with cirrhosis: a randomized, controlled trial. Gastroenterology 2014;147(6):1327-37. 